\title{
Comparison of the physiological properties of human periodontal-masseteric reflex evoked by incisor and canine stimulation
}

\author{
Hiroko Ohmori *, Hiroaki Kirimoto and Takashi Ono \\ Orthodontic Science, Graduate School, Tokyo Medical and Dental University, Tokyo, Japan
}

\section{Edited by:}

Sachiko Iseki, Tokyo Medical and Dental University, Japan

Reviewed by:

Ariane Berdal, University

Paris-Diderot, France

Mario De Rosa, Second University of Naples, Italy

Kazuo Toda, Nagasaki University,

Japan

${ }^{*}$ Correspondence:

Hiroko Ohmori, Orthodontic Science,

Graduate School, Tokyo Medical and

Dental University, 1-5-45 Yushima,

Bunkyo-ku, Tokyo 113-8549, Japan.

e-mail:ohmori.orts@tmd.ac.jp

Part of this material was presented at the 87th Congress of the European

Orthodontic Society, Istanbul, Turkey,

June 19-23, 2011.

\begin{abstract}
Introduction: The present study was designed to clarify whether the bilateral cooperation in the human periodontal-masseteric reflex (PMR) differs between central incisors and canines. Methods: Surface array electrodes were placed on the bilateral masseter muscles to simultaneously record the firing activities of single motor units from both sides in seven healthy adults. During light clenching, mechanical stimulation was applied to the right maxillary central incisor and canine to evoke the PMR. Unitary activity was plotted with respect to the background activity and firing frequency. The slope of the regression line (SRL) and the correlation coefficient (CC) between the central incisor and canine and the lateral differences between these values were compared. Results: There were significant differences in the $S R L$ and $C C$, as well as lateral differences, between the central incisor- and canine-driven PMR. Discussion: These results suggest that the PMR differs depending on both the tooth position and laterality.
\end{abstract}

Keywords: periodontal-masseteric reflex, mechanoreceptor, motor unit, teeth, human

\section{INTRODUCTION}

Jaw movement during mastication exhibits a rhythmic pattern controlled by a central mechanism (Delcomyn, 1980; Morquette et al., 2012). Although experiments on animals have established the existence of a pattern generator for mastication (Dellow and Lund, 1971), there is evidence that masticatory forces are precisely controlled by peripheral feedback and that these forces change from bite to bite depending on the consistency of the bolus (Lund, 1991; Thexton, 1992; Türker, 2002) and the required task (Farella et al., 2009).

It is widely accepted that there are many receptors, temporomandibular joint receptors, muscle spindles, skin, and mucosal receptors including the periodontal mechanoreceptors (reviewed in Lund, 1991). Among them, afferent information regarding forces acting on the teeth is important for the sensorimotor regulation of mastication (reviewed in Lund, 1991; also see Türker et al., 2007). The force-encoding properties of periodontal afferents that supply anterior teeth have been described in several animal species (Hannam, 1982; Linden, 1990). While some studies have also been performed in humans, they only targeted the incisors (Türker et al., 1994, 1997; Trulsson and Johansson, 1996; Yang and Türker, 2001; Brinkworth et al., 2003; Sowman

Abbreviations: BGA, background MU activity; CC, correlation coefficient; EMG, electromyography; FR, firing rate; $\mathrm{MU}$, motor unit; MVC, maximum voluntary contraction; PMR, periodontal-masseteric reflex; RR, reflex response; sRL, slope of the regression line. et al., 2007, 2010; Sowman and Türker, 2008; Naser-ud-Din et al., 2010).

With regard to function, different classes of teeth have different shapes (Lucas, 2004). Indeed, among the anterior teeth, the incisors, and canines play different roles in mastication in humans. The periodontal-masseteric reflex (PMR) has historically been used to understand the role of intra-oral mechanoreceptors in masticatory function, and the central incisor-driven PMR has been studied exclusively (Sessle and Schmitt, 1972; Louca et al., 1994; Türker et al., 1994; Türker and Jenkins, 2000; Sowman and Türker, 2008), while involvement of the canines has not been studied. Moreover, the mechanism that underlies the bilateral cooperation of the PMR remains unclear, although the PMR is evoked bilaterally even under unilateral stimulation. Another potential shortcoming in previous studies that used electromyographic (EMG) recording to study the PMR is the use of mass EMG potentials (Türker et al., 1997; Brinkworth et al., 2003; Sowman and Türker, 2008; Naser-ud-Din et al., 2010). Although an analysis of mass EMG potentials can provide ample information regarding the global tendency of muscle activity, small changes at the functional level of motor units (MU) may not be detected (Türker et al., 1994). In a previous study, we reported that the asymmetrical reflexive MU response was evoked by stimulation of the canine using a surface array electrode (Ohmori et al., 2009b). Unfortunately, we did not compare canine- and central incisor-driven PMRs in that study. 
Thus, the present study was designed to clarify whether there is a functional difference in the sensory inputs from periodontal mechanoreceptors innervated in the incisor and canine with special attention to the properties of the MU response.

\section{MATERIALS AND METHODS SUBJECTS}

Experiments were conducted on seven male volunteers aged 28-36 years [mean \pm standard deviation (SD): $31 \pm 3.0$ years], all of whom gave their written informed consent. The study was approved by the University of Tokyo Medical and Dental University Human Ethics Committee and conformed to the Declaration of Helsinki. All subjects had a full complement of natural teeth, without malocclusion, except for the absence of the third molars. Subjects were excluded from this study if they had any acute or chronic injury or systemic disease, such as acute pain, that could interfere with the outcome; chronic pain, clinical pathology, or previous surgery related to the masticatory system; or if they complained of symptoms of temporomandibular disorders before the test. None of the subjects had any neurological problems in their medical history, and none were taking medication specifically intended to affect the musculoskeletal system, such as anti-inflammatory or pain-relief drugs, muscle relaxants, or arthritic medications.

During the experimental sequences, the subjects were seated upright in a chair adjusted for height so that they could bite onto fixed metal bite plates. The device was similar to one described previously (Ohmori et al., 2009b). The metal bite plates were coated with a dental impression material that molded to each subject's teeth. The subjects bit into silicone putty material (Exafine Putty Type, GC Corp., Tokyo, Japan) to produce impressions of their maxillary and mandibular teeth. The impressions were recorded such that the maxillary and mandibular incisors were held comfortably apart by approximately $6 \mathrm{~mm}$. The upper peri-incisal part of this impression was trimmed so that both the right maxillary incisor and canine were fully exposed. The impression was secured to a U-shaped metal bar, which was rigidly attached to a table.

\section{ELECTROMYOGRAPHIC RECORDING}

Surface array electrodes (Figure 1A; TOG206-036; Unique Medical Co., Ltd., Komae, Tokyo, Japan; Ag/Ag-Cl, diameter: 1.0 mm, interelectrode distance: $5 \mathrm{~mm}$ ) were placed on the bilateral masseter muscles to simultaneously record the firing activities of single MUs from both sides (Ohmori et al., 2009a). The skin was prepared by rubbing with gauze soaked in alcohol. The electrode was placed to be as close as possible, and to be parallel to the direction of the muscle fiber in the area between the center of the masseter muscle and the gonion (the most lateral point of the mandibular angle, by palpation) to avoid the influence of the buccinator muscle and other facial muscles. Four or five recordings were performed on different sites of each respective muscle. MUs that fired in response to a small occlusal force were selected visually to control the firing rates (FRs); e.g., the buccinator muscle pulls the angles of the mouth laterally as an antagonist to the muscles of protrusion and rounding. The time constant of the amplifier was set at $0.03 \mathrm{~s}$; potential was measured using adjoining selected bipolar electrodes. Differential motion was amplified to two simultaneous channels that measured MU (AB-621G; Nihon Kohden, Tokyo, Japan; $\times 1000$ gain; $100 \mathrm{~Hz}$ and $1 \mathrm{kHz}$ for low- and high-pass filters, respectively). A reference electrode was placed on the subject's left earlobe.

The maximum voluntary contraction (MVC) was first determined, and, during experiments, particular levels of contraction were sustained with the aid of a visual feedback device (Figure 1B). The subject was instructed to lightly clench (i.e., 10\% of MVC) with visual feedback for $1 \mathrm{~min}$.

\section{MECHANICAL TOOTH STIMULATION}

The subjects wore a cheek retractor throughout the experimental period, so that the experimenter could stimulate the specific targeted intra-oral region without touching the surrounding structures. During clenching, mechanical stimulation with a trapezoidal wave at $0.5 \mathrm{~N}$, with a $100-\mathrm{ms}$ rise time and a total duration of $2.2 \mathrm{~s}$, was applied in a ramp-and-hold fashion to the right maxillary central incisor and canine linguolabially and orthogonally to evoke the PMR via a probe (Mechanical Stimulator, Diamedical Inc., Tokyo, Japan). The mechanical stimulation was randomized to start. The subject was instructed to view the monitor screen to control his clenching level, so that the subject could not anticipate the timing of the delivery of mechanical stimulation. The task consisted of 10-s rest and 2-s stimulation periods that were alternately repeated four times. During data acquisition, the subject was instructed to breathe through the nose naturally.

\section{DATA ANALYSIS}

The data were converted from an analog to a digital signal at a sampling frequency of $5 \mathrm{kHz}$ (CED 1401; Cambridge Electronic Design, Inc., Cambridge, UK), and stored for further analysis using Spike2 software (Cambridge Electronic Design, Inc.). MUs were discriminated offline using the Spike2 program, and units were separated using the program's template matching algorithm. The mean FR was calculated from data on the MU activity for $1 \mathrm{~s}$ during $2.2 \mathrm{~s}$ of stimulation and the background MU activity (BGA) during $1 \mathrm{~s}$ before stimulation, and the differences between these values [reflex response (RR)] were compared. However, transitionary responses were excluded from when stimulation started and stopped.

Statistical analysis was performed using StatView software (SAS Institute, Cary, NC, USA). The normality of data was examined using a Jarque-Bera test. The statistical significance of differences between the central incisor and canine, as well as between the right (i.e., ipsilateral) and left (i.e., contralateral) sides, was determined with a paired Student's $t$-test. Unitary activity was plotted with respect to the FR and BGA to obtain both a regression line and correlation coefficient (CC). The CC was compared using Fisher's $Z$ test. The slope of the regression line (sRL) and the $x$-intercept for the central incisor and canine, as well as those for the right and left sides, were compared using Student's $t$-test. Values of $p<0.05$ were considered significant.

\section{RESULTS}

From the seven subjects, 32 pairs of MUs were recorded from the right and left masseter muscles that responded to mechanical stimulation. Patterns of MU activity for the 32 pairs are summarized 


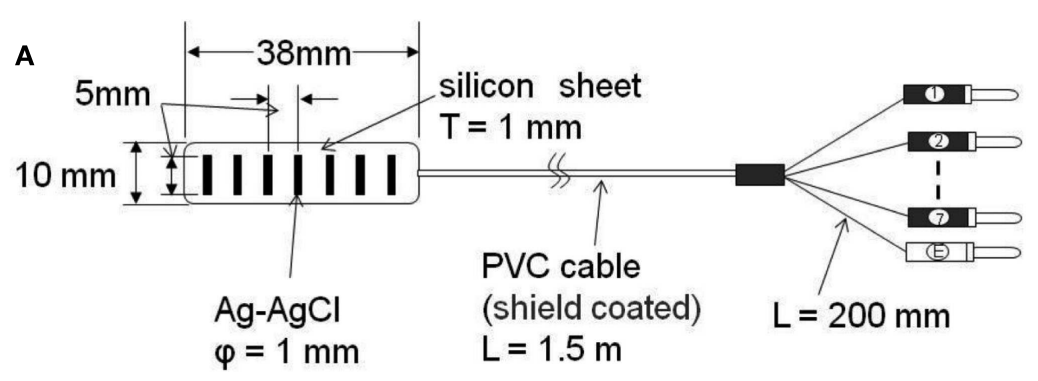

B

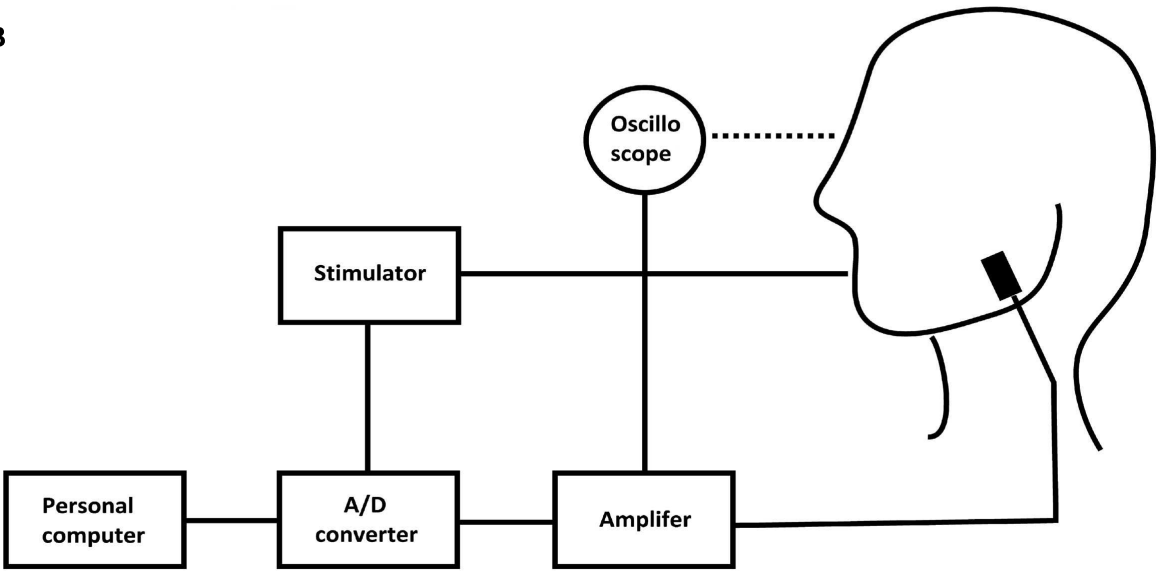

FIGURE 1 | Schematic illustration of the electrode (A) and recording diagram for the PMR (B). Abbreviations: T, thick; $\varphi$, diameter; L, long; $\mathrm{Ag}$, silver; $\mathrm{Ag}-\mathrm{Cl}$, silver-chloride; PVC, polyvinyl chloride; A/D, analog-digital.

in Table 1. There were no significant differences in the mean FR, $\mathrm{BGA}$, or RR between the right and left masseter muscles. With regard to the receptive field, the mean FR for the canine was significantly higher than that for the central incisor. The mean RR for the canine was significantly higher than that for the central incisor.

A typical record of the canine-driven PMR in the bilateral masseter muscles under mechanical stimulation is shown in Figure 2A. Superimposition of the MU activity recorded from the both masseter muscle revealed that the duration, amplitude, and shape are identical, indicating that the activity was recorded from a single MU. For the left masseter MU, the BGA was $12 \mathrm{~Hz}$ and the FR increased to $14 \mathrm{~Hz}$ during stimulation and decreased to the original BGA after the cessation of stimulation.

The relationship between the BGA and RR for the central incisor- and canine-driven PMRs is shown in Figure 2B. The formulae for the regression lines for the response are Central incisor: $y=-0.902 x+11.1$ on the right side, and $y=-0.863 x+9.94$ on the left side Canine: $y=-0.458+7.63$ on the right side, and $y=-0.684 x+9.67$ on the left side where $x$ and $y$ denote the BGA $(\mathrm{Hz})$ and $\mathrm{RR}(\mathrm{Hz})$, respectively.

From these regression lines, the $x$-intercepts were obtained (Figure 2B). These points correspond to the threshold of excitatory and inhibitory effects. The thresholds for the central incisor were $12.3 \mathrm{~Hz}$ on the right side and $11.5 \mathrm{~Hz}$ on the left side, and those for the canine were $16.7 \mathrm{~Hz}$ on the right side and $14.1 \mathrm{~Hz}$ on the left side. The threshold for the canine-driven PMR was significantly $(p<0.05)$ larger than that for the central incisor-driven PMR. Regarding laterality, there was no significant difference in the threshold for the central incisor-driven PMR. However, for the canine-driven PMR, the threshold for the right masseter muscle was significantly $(p<0.05)$ larger than that for the left masseter muscle. Therefore, the excitatory reflex was easily evoked at less than $11 \mathrm{~Hz}$, and the inhibitory reflex was easily evoked at more than $17 \mathrm{~Hz}$ for both the central incisor and canine. The CC for the comparisons between the BGA and RR for the central incisor was -0.90 for the right masseter muscle and -0.85 for the left masseter muscle, and that for the canine was -0.62 for the right masseter muscle and -0.81 for the left masseter muscle. The correlation on the ipsilateral side was significantly $(p<0.05)$ weaker than that on the contralateral side for the canine, but not the central incisor.

Regarding the receptive field, the $s R L$ was significantly $(p<0.05)$ steeper and the negative CC was significantly $(p<0.05)$ greater for the central incisor-driven PMR than for the caninedriven PMR. The negative $\mathrm{CC}$ for the contralateral caninedriven PMR was significantly $(p<0.05)$ greater than that for the ipsilateral one.

\section{DISCUSSION}

To date, only a few studies have investigated the PMR responses of single MUs in humans. However, they used invasive intramuscular fine-wire electrodes and only studied central incisor-driven PMR unilaterally (Türker et al., 1994; Yang and Türker, 2001; Sowman et al., 2007). Surface electrodes are a feasible approach for non-invasively studying MU discharge patterns (Sun et al., 2000; Zwarts and Stegeman, 2003). Therefore, this is the first study to 
Table 1 | Comparison of FR, BGA, and RR for central incisor- and canine-driven PMRs.

\begin{tabular}{llllr}
\hline Receptive field & Side & FR (Hz) & BGA (Hz) & RR (Hz) \\
\hline Central incisor & Ipsi & $12.2 \pm 1.20$ & $10.7 \pm 2.66$ & $1.45 \pm 2.67$ \\
& Contra & $11.4 \pm 1.23^{*}$ & $10.3 \pm 2.23$ & $1.05 \pm 2.26^{\dagger}$ \\
Canine & Ipsi & $13.4 \pm 2.37$ & $10.7 \pm 2.99$ & $2.72 \pm 2.21$ \\
& Contra & $13.2 \pm 2.75^{*}$ & $11.1 \pm 2.75$
\end{tabular}

Data are presented as the means \pm standard deviations (SDs). * The mean FR for the canine was significantly higher than that for the incisor; ${ }^{\dagger}$ the mean $R R$ for the canine was significantly higher than that for the central incisor. There were no significant differences in the mean FR, BGA, or RR between the right and left masseter muscles.

$F R$, firing rate; $B G A$, background activity; $R R$, reflex response.

\section{A}

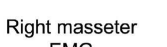

EMG

Left masseter

EMG

Left masseter

(Firing rate)

Stimulation
(right)
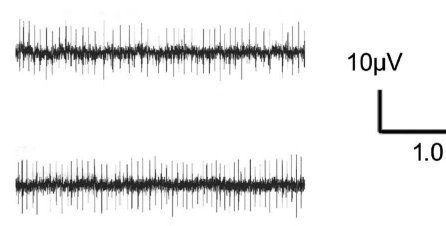

$1.0 \mathrm{~s}$
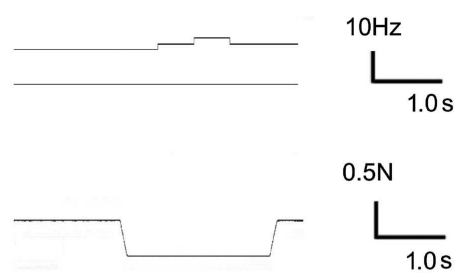

B

\section{Right incisor}

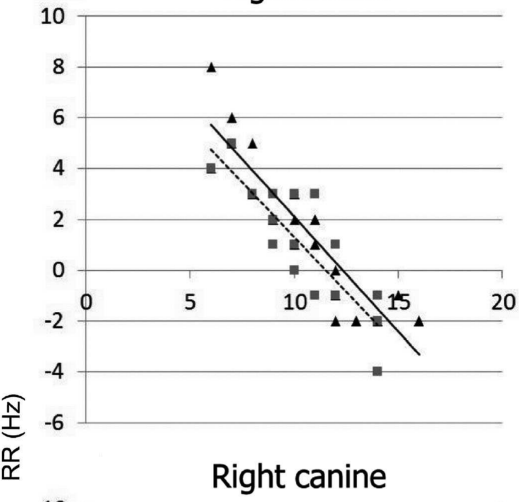

Right canine

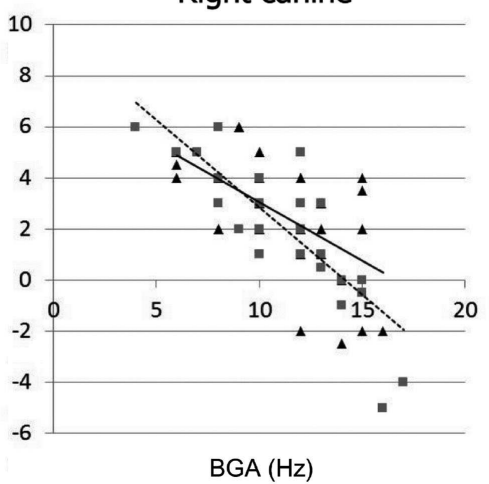

FIGURE 2 | (A) Typical canine-driven PMR (B), Scatter plots and corresponding regression lines of the background activity (BGA; $x$-axis), and the reflex response (RR; $y$-axis). Triangle, motor unit of the right masseter muscle; square, motor unit of the left masseter muscle.

use a non-invasive surface electrode to examine the functional differences between the central incisor- and canine-driven PMRs and their laterality at the level of single MU. Thus, we believe that these findings represent an important contribution to the literature.

Mandibular movement is modified by feedback from the periodontal membrane (Takada et al., 1996; Türker et al., 2007). It has been shown that the change from an excitatory reflex to an inhibitory reflex is related to the size of MUs, BGA, or the direction and strength of the mechanical stimulation applied to the teeth (Trulsson and Johansson, 1996; Türker et al., 1997; Yang and Türker, 2001; Brinkworth and Türker, 2005). When axial pressure stimulation was applied to the maxillary molar, an inhibitory reflex occurred in the masseter muscle when the occlusal force was large, while an excitatory reflex was easily evoked when the occlusal force was small (Yamamura et al., 1993). Sowman and Türker (2008) found that the RR of incisor-driven PMR is negatively correlated with the amount of pre-load applied to the incisor. Our finding that there was a significant negative correlation between $R R$ and BGA for the canine as well as the central incisor is consistent with their findings.

Lingobuccal and orthogonal mechanical stimulation of the upper central incisor or canine caused an inhibitory reflex at high BGA and an excitatory reflex at low BGA. With an increase in the BGA, the reflex was reversed from excitatory to inhibitory. These findings were similar to those in our previous study (Ohmori et al., 2009b), and the dependency of the RR on the BGA suggests 


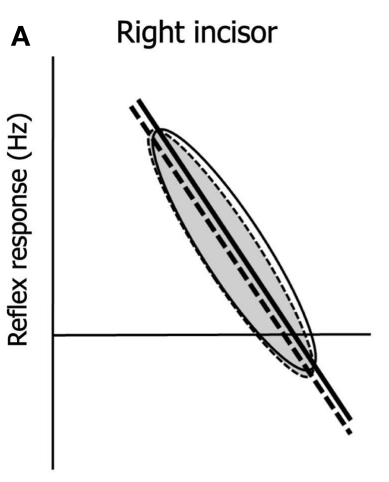

FIGURE 3 | Schematic illustrations of the central incisor- and canine-driven PMRs which show the dependence of the reflex response on the background activity. The ellipses are a conceptual explanation for the correlation coefficient. (A) Right (solid line) and left (dashed line) central incisor- (a) and canine-driven PMRs (b); (B) comparison of the central incisor
Right canine

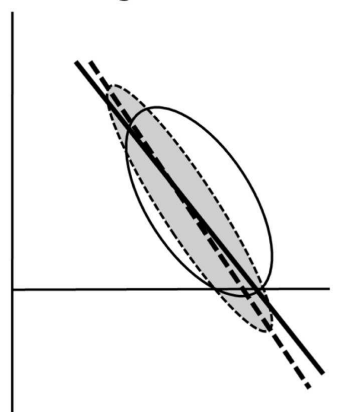

Background Activity $(\mathrm{Hz})$
B Right incisor/canine

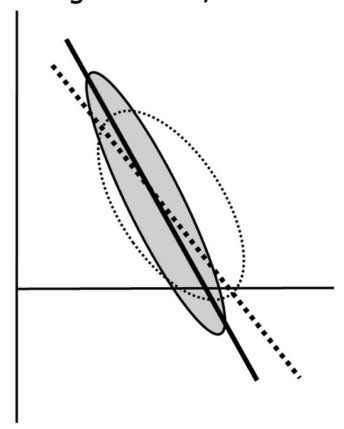

(solid line)- and canine (dotted line)-driven PMRs. Abbreviations: BGA, background MU activity; CC, correlation coefficient; EMG, electromyography; $F R$, firing rate; $M U$, motor unit; MVC, maximum voluntary contraction; PMR, periodontal-masseteric reflex; $R R$, reflex response; $s R L$, slope of the regression line. the existence of fine motor control of the jaw-closing muscle. Although there was a significant difference in the BGA threshold for reversal of the RR between the central incisor and canine, the functional significance of the actual value and the difference is not yet clear.

Although there was no difference in the BGA, there was a difference in the reflex effect between sides for the canine (Figure 3A). Kamata and colleagues reported a RR in the ipsilateral and contralateral temporal muscles when the canine was mechanically stimulated (Kamata, 1994; Kamata et al., 1994, 1995). Either the input from the canine periodontal mechanoreceptor strongly affects the contralateral side, or the change in the FR of the masseter muscle influences the feedback mechanism via bilateral cooperation. On the other hand, there was no difference between sides for the central incisor (Figure 3A). These findings suggest that the canine may control lateral jaw movement to a greater degree than does the central incisor, and that input from the canine may have a greater effect on lateral movement than that from the central incisor.

The functional difference between the central incisor- and canine-driven PMRs is shown in Figure 3B. This suggests that the $\mathrm{RR}$ is more sensitive to the change in BGA for the central incisor than for the canine. Moreover, the difference in the laterality of CC between the BGA and RR for the central incisor and canine suggests that there may be a functional difference between the central incisor and canine with respect to the information received from periodontal mechanoreceptors regarding orthogonal displacement: e.g., the incisors cut the food bolus vertically, so that they have little horizontal function. In contrast, the canines play a guiding role during lateral jaw movement. The

\section{REFERENCES}

Brinkworth, S. A., and Türker, K. S. (2005). Jaw movement alters the reaction of human jaw muscles to incisor stimulation. Exp. Brain Res. 164, $165-176$.
Brinkworth, S. A., Türker, K. S., and Savundra, A. W. (2003). Response of human jaw muscles to axial stimulation of the incisor. J. Physiol. (Lond.) 547, 233-245.

Delcomyn, F. (1980). Neural basis of rhythmic behavior inferior alveolar nerves were more sensitive to the central incisor stimulation than the canine (Trulsson and Essick, 2010). Thus, periodontal mechanoreceptors of the central incisors may carry more information than those of the canines, or the canine-driven PMR may be more strongly influenced by other proprioceptive factors from intra- and juxta-oral organs: e.g., the temporomandibular joint, muscle spindles, and tongue (Takada et al., 1996).

Even though the masseter and temporalis muscles are both jawclosers, the incisor-driven activation patterns are different: there are common synaptic inputs to the motor nucleus of the left and right masseter, but not to the left and right temporalis (Jaberzadeh et al., 2006). Our present findings add a further interpretation of their study in that this was the case for central incisor-driven, but not canine-driven activation of the masseter muscle. This indicates that even in homologous muscle pairs, the peripherally driven activation pattern differs depending on the receptive field. Sowman et al. (2010) reported that the threshold for the detection of incisal forces was changed by jaw position during jaw movement. The modulation of masticatory reflexes of PMR origin needs to be studied in conscious humans during mastication, or at least under conditions where automatic, rhythmic movements that approximate mastication are being performed (Türker et al., 2007). In other jaw conditions, further studies are needed to make the reflexive modulation for mastication more obvious.

\section{ACKNOWLEDGMENTS}

We would like to thank Prof. Emeritus Kunimichi Soma of Tokyo Medical and Dental University for his initial guidance and suggestions regarding this experiment. 
Hannam, A. G. (1982). "The innervations of the periodontal ligament," in The Periodontal Ligament in Health and Disease eds B. K. B. Berkovitz, B. J. Moxham and H. N. Newman (Oxford: Pergamon Press), 173-196.

Jaberzadeh, S., Miles, T. S., and Nordstrom, M. A. (2006). Organisation of common inputs to motoneuron pools of human masticatory muscles. Clin. Neurophysiol. 117, 1931-1940.

Kamata, S. (1994). Reflex response of temporal muscle induced by mechanical stimulation to periodontal ligament - in the lateral jaw movement during mastication. Kokubyo Gakkai Zasshi 61, 82-97.

Kamata, S., Fujita, Y., and Soma, K. (1995). Reflex response of temporal muscle induced by mechanical stimulation of human canine - comparison of normal overjet and cross bite responses. J. Jpn. Soc. Stomatognathic Funct. 1, 281-287.

Kamata, S., Fujita, Y., Toda, K., and Soma, K. (1994). Excitatory reflex response of contralateral temporal muscle evoked by mechanical stimulation at human maxillary canine. J. Jpn. Soc. Stomatognathic Funct. 1, 127-132.

Linden, R. W. A. (1990). Neurophysiology of the Jaws and Teeth. New York: Macmillan.

Louca, C., Cadden, S. W., and Linden, R. W. A. (1994). Inhibitory jaw reflexes and the role played by periodontal ligament mechanoreceptors. J. Dent. Res. 73, 791.

Lucas, P. W. (2004). Dental Functional Morphology: How Teeth Work. Cambridge: Cambridge University Press.
Lund, J. P. (1991). Mastication and its control by the brain stem. Crit. Rev. Oral Biol. Med. 2, 33-64.

Morquette, P., Lavoie, R., Fhima, M. D., Lamoureux, X., Verdier, D., and Kolta, A. (2012). Generation of the masticatory central pattern and its modulation by sensory feedback. Prog. Neurobiol. 96, 340-355.

Naser-ud-Din, S., Sowman, P. F., Dang, H., and Türker, K. S. (2010). Modulation of masseteric reflexes by simulated mastication. J. Dent. Res. 89, 61-65.

Ohmori, H., Kirimoto, H., and Soma, K. (2009a). Characteristics and clinical application of a surface array electrode for recording masticatory muscle motor unit action potentials. Orthod. Waves 68, 57-63.

Ohmori, H., Kirimoto, H., and Soma, K. (2009b). Bilateral asymmetries in periodontal-masseteric reflex activity in man. Orthod. Waves 68, 147-151.

Sessle, B. J., and Schmitt, A. (1972). Effects of controlled tooth stimulation of jaw muscle activity in man. Arch. Oral Biol. 17, 1597-1607.

Sowman, P. F., Brinkworth, R. S., and Türker, K. S. (2010). Threshold for detection of incisal forces is increased by jaw movement. J. Dent. Res. 89, 395-399.

Sowman, P. F., Ogston, K. M., and Türker, K. S. (2007). Periodontal anaesthetization decreases rhythmic synchrony between masseteric motor units at the frequency of jaw tremor. Exp. Brain Res. 179, 673-682.

Sowman, P. F., and Türker, K. S. (2008). Periodontal-masseteric reflexes decrease with tooth pre-load. J. Dent. Res. 87, 175-179.
Sun, T. Y., Chen, J. J., and Lin, T. S. (2000). Analysis of motor unit firing patterns in patients with central or peripheral lesions using singular-value decomposition. Muscle Nerve 23, 1057-1068.

Takada, K., Yashiro, K., Sorihashi, Y. Morimoto, T., and Sakuda, M. (1996). Tongue, jaw, and lip muscle activity and jaw movement during experimental chewing efforts in man. J. Dent. Res. 75, 1598-1606.

Thexton, A. J. (1992). Mastication and swallowing: an overview. Br. Dent. J. 173, 197-206.

Trulsson, M., and Essick, G. K. (2010) Sensations evoked by microstimulation of single mechanoreceptive afferents innervating the human face and mouth. J. Neurophysiol. 103, 1741-1747.

Trulsson, M., and Johansson, R. S. (1996). Encoding of tooth loads by human periodontal afferents and their role in jaw motor control. Prog. Neurobiol. 49, 267-284.

Türker, K. S. (2002). Reflex control of human jaw muscles. Crit. Rev. Oral Biol. Med. 13, 85-104.

Türker, K. S., Brodin, P., and Miles, T. S. (1994). Reflex responses of motor units in human masseter muscle to mechanical stimulation of a tooth. Exp. Brain Res. 100, 307-315.

Türker, K. S., and Jenkins, M. (2000). Reflex responses induced by tooth unloading. J. Neurophysiol. 84 1088-1092.

Türker, K. S., Sowman, P. F., Tuncer, T., Tucker, K. J., and Brinkworth, R. S. (2007). The role of periodontal mechanoreceptors in mastication. Arch. Oral Biol. 52, 361-364.

Türker, K. S., Yang, J., and Brodin, P. (1997). Conditions for excitatory or inhibitory masseteric reflexes elicited by tooth pressure in man. Arch. Oral Biol. 42, 121-128.

Yamamura, C., Kosugi, S., Ono, K., and Shimada, K. (1993). Patterns of jaw reflexes induced by incisal and molar pressure stimulation in relation to background levels of jaw-clenching force in humans. Jpn. J. Physiol. 43, 87-102.

Yang, J., and Türker, K. S. (2001). Distribution of periodontal afferent input to motoneurons of human masseter. Arch. Oral Biol. 46, 989-996.

Zwarts, M. J., and Stegeman, D. F. (2003). Multichannel surface EMG: basic aspects and clinical utility. Muscle Nerve 28, 1-17.

Conflict of Interest Statement: The authors declare that the research was conducted in the absence of any commercial or financial relationships that could be construed as a potential conflict of interest.

Received: 10 April 2012; paper pending published: 06 May 2012; accepted: 11 June 2012; published online: 28 June 2012.

Citation: Ohmori H, Kirimoto $H$ and Ono T (2012) Comparison of the physiological properties of human periodontalmasseteric reflex evoked by incisor and canine stimulation. Front. Physio. 3:233. doi: 10.3389/fphys.2012.00233

This article was submitted to Frontiers in Craniofacial Biology, a specialty of Frontiers in Physiology.

Copyright ( $\odot 2012$ Ohmori, Kirimoto and Ono. This is an open-access article distributed under the terms of the Creative Commons Attribution Non Commercial License, which permits noncommercial use, distribution, and reproduction in other forums, provided the original authors and source are credited. 